\title{
Gait patterns in COPD: the Rotterdam Study
}

\author{
Lies Lahousse ${ }^{1,2,9}$, Vincentius J.A. Verlinden ${ }^{2,3,9}$, Jos N. van der Geest ${ }^{4}$, \\ Guy F. Joos ${ }^{1}$, Albert Hofman ${ }^{2,5}$, Bruno H.C. Stricker ${ }^{2,5,6,7}$, Guy G. Brusselle ${ }^{1,2,8}$ \\ and M. Arfan Ikram $2,3,4$
}

\begin{abstract}
Affiliations: ${ }^{1}$ Dept of Respiratory Medicine, Ghent University Hospital, Ghent, Belgium. ${ }^{2}$ Dept of Epidemiology, Erasmus Medical Center, Rotterdam, The Netherlands. ${ }^{3}$ Dept of Radiology, Erasmus Medical Center, Rotterdam, The Netherlands. "Dept of Neuroscience, Erasmus Medical Center, Rotterdam, The Netherlands. ${ }^{5}$ Members of the Netherlands Consortium on Healthy Aging, Leiden, The Netherlands. ${ }^{6}$ Dept of Medical Informatics, Erasmus Medical Center, Rotterdam, The Netherlands. ${ }^{7}$ Inspectorate of Healthcare, The Hague, The Netherlands. ${ }^{8}$ Dept of Respiratory Medicine, Erasmus Medical Center, Rotterdam, The Netherlands. ${ }^{9}$ These authors contributed equally.
\end{abstract}

Correspondence: Bruno H.C. Stricker, Dept of Epidemiology, Erasmus University Medical Center, PO Box 2040, 3000 CA Rotterdam, The Netherlands. E-mail: b.strickerdaerasmusmc.nl

ABSTRACT Gait disturbances in patients with chronic obstructive pulmonary disease (COPD) may lead to disability and falls. As studies assessing gait kinematics in COPD are sparse, we investigated associations of COPD with various gait domains and explored a potential link with falling.

Gait was measured within the prospective, population-based Rotterdam Study (age $\geqslant 55$ years) using an electronic walkway and summarised into seven gait domains: Rhythm, Variability, Phases, Pace, Tandem, Turning and Base of Support. Rhythm is a temporal gait aspect that includes cadence and reflects how quickly steps are taken.

Persons with COPD ( $\mathrm{n}=196)$ exhibited worse Rhythm ( -0.21 SD, 95\% CI $-0.36--0.06 \mathrm{SD})$ compared with persons with normal lung function $(n=898)$, independent of age, sex, height, education, smoking or analgesic use, especially when dyspnoea and severe airflow limitation or frequent exacerbations (Global Initiative for Chronic Obstructive Lung Disease group D: $-0.83 \mathrm{SD}, 95 \% \mathrm{CI}-1.25--0.41 \mathrm{SD}$ ) were present. A lower forced expiratory volume in $1 \mathrm{~s}$ was associated with worse Rhythm and Pace, including lower cadence and gait velocity, respectively. Importantly, fallers with COPD had significantly worse Rhythm than nonfallers with COPD.

This study demonstrates that persons with COPD exhibit worse Rhythm, especially fallers with COPD. The degree of Rhythm deterioration was associated with the degree of airflow limitation, symptoms and frequency of exacerbations.

@ERSpublications

This study comprehensively investigates, in a large, population-based cohort, the gait pattern in COPD http://ow.ly/HQbc3

This article has supplementary material available from erj.ersjournals.com

Received: June 272014 | Accepted after revision: Dec 272014 | First published online: Feb 192015

Support statement: This study was supported by the Fund for Scientific Research Flanders (FWO) project G035014N, and the Netherlands Organization for Scientific Research (NWO) grants 904-61-093 and 918-46-615. Lies Lahousse is the recipient of a European Respiratory Society fellowship (Short-Term Research Training Fellowship number 1312011). The funding source had no involvement in data collection, analysis, writing, data interpretation or the decision to submit the paper for publication. Funding information for this article has been deposited with FundRef.

Conflict of interest: Disclosures can be found alongside the online version of this article at erj.ersjournals.com

Copyright OERS 2015 


\section{Introduction}

Worldwide, chronic obstructive pulmonary disease (COPD) is a leading cause of morbidity and mortality [1]. COPD is primarily characterised by persistent airflow limitation that is associated with an abnormal inflammatory response of the lungs to noxious particles or gases [2]. However, its systemic effects also have an important impact on symptoms and prognosis [1]. Even in COPD patients with mild disease, cardiovascular comorbidities and muscle weakness precede the development of functional limitations [3]. As COPD also affects function and mobility, persons with COPD have an increased risk of falling [3-8]. Falling is a major health concern, representing one of the main causes of pain, disability and death in the elderly $[7,9]$. Even without injuries, a fall can negatively impact the quality of life of an individual through fear of falling, which often inhibits performance of activities [10].

Although causes of falls are often multifactorial, the majority of falls occurs during walking and gait analysis may be the best tool to predict the first fall onset [11, 12]. Poor gait is indeed one of the main risk factors of falls and an important indicator of general health [13-15]. Gait changes are often too subtle to be detected by clinical observation alone; however, clinical consequences of such subtle changes may be considerable [11]. Gait is affected by various organ systems, such as the central nervous system, cardiovascular system and musculoskeletal system, and poor gait is a strong risk factor of death [16-20]. COPD may affect gait through any of these systems.

Gait is a complex concept that can be assessed using many parameters, which can be summarised into seven gait domains [19]. To date, COPD has mostly been associated with a shorter walking distance during the well validated 6-min walk test. Associations of COPD with specific gait domains may give an indication of the pathways underlying their associations with gait. In turn, this knowledge may aid in identifying better intervention strategies to prevent future falling in patients with COPD.

The aim of this study was to investigate the hypothesis that both spatiotemporal and balance aspects of gait are worse in persons with COPD compared with persons with normal lung function. Moreover, we evaluated the influence of COPD severity by airflow limitation, symptoms and/or frequent exacerbations. Additionally, we explored a potential link between gait differences in COPD and falling.

\section{Methods}

\section{Study design and COPD assessment}

The present study is embedded within the population-based Rotterdam Study (described in the online supplementary material) and comprises all participants who completed gait assessment and spirometry successfully up to December 2011 [21]. Spirometry was performed using a Master Screen PFT Pro (CareFusion, San Diego, CA, USA) by trained paramedical personnel according to the American Thoracic Society/European Respiratory Society guidelines [22, 23]. Participants with asthma or spirometry suggestive of a restrictive syndrome were excluded. The diagnosis and classification of COPD was based on the Global Initiative for Chronic Obstructive Lung Disease (GOLD) criteria (proportion of the forced vital capacity (FVC) exhaled in the first second (forced expiratory volume in $1 \mathrm{~s}(\mathrm{FEV} 1) / \mathrm{FVC}$ ratio) $<70 \%$ ) and classified as mild or moderate/severe airflow limitation by $\mathrm{FEV}_{1} \geqslant 80 \%$ or $<80 \%$ predicted, respectively $[24,25]$. Furthermore, participants with COPD were also classified according to the updated GOLD group categorisation into group A (low risk and fewer symptoms), B (low risk but more symptoms), C (high risk but fewer symptoms) or D (high risk and more symptoms) [1]. Exacerbations were counted as the total number of moderate and severe exacerbations in the year 2010. Frequent exacerbators were defined as persons with COPD having at least two moderate or severe exacerbations.

\section{Assessment of gait}

Gait was assessed with a $5.79-\mathrm{m}$-long electronic walkway with $1.27-\mathrm{cm}$-wide pressure sensors $(4.88 \times 0.61-\mathrm{m}$ active area) (GAITRite Platinum; CIR systems Inc., Sparta, NJ, USA) [19]. The walkway checked for activation of the pressure sensors at $120 \mathrm{~Hz}$. The standardised gait protocol includes three different walking conditions (fig. 1): "normal walk", "turn" and "tandem walk". In normal walk, participants were asked to walk at their usual pace across the walkway, until they were off the walkway. The normal walk was performed four times in both directions (eight recordings). In turn, participants were asked to walk at their usual pace across the walkway, turn halfway and return to their starting position (one recording). In tandem walk, participants were asked to walk tandem (heel to toe) along a visible line on the walkway until they were off the walkway (one recording). The first recording of the normal walks was considered a practice walk and not included in the analyses.

All gait parameters from the normal walk were automatically quantified by the walkway software. Parameters were quantified for both steps and strides (e.g. step length is the distance between two opposite footsteps on the line of progression, while stride length is the distance between two footsteps on the same side of the line of progression). Variability measures were quantified as standard deviations of the gait 
parameters among steps or strides. Turning time was calculated from the walkway-generated data as the time between foot-off of the first footstep and first contact of the last footstep (fig. 1). Turning step count was calculated as the automatically quantified number of steps in the turn minus one. Errors in tandem walking were calculated as the distance of sidesteps from the line and the surface of these sidesteps, with aid of the automatically generated surface and distance parameters from the walkway software. Additionally, we scored the number of double steps, i.e. two consecutive steps with the same foot on the line, as errors in tandem walking.

As previously described, principal component analysis was used to summarise 30 gait parameters, including 25 from normal walking, two from turning and three from tandem walking, into fewer independent gait domains, while capturing the largest amount of variance. Each gait domain had to explain at least as much variance as a single gait parameter. Varimax rotation was used to ensure that the gait domains were mutually independent. We found seven independent gait domains: Rhythm, Variability, Phases, Pace, Tandem, Turning and Base of Support [19]. Among others, Rhythm reflects cadence and stride time; Variability reflects stride length and stride time variability; Phases reflects double support time and double support as a percentage of the gait cycle; Pace reflects stride length and velocity; Tandem reflects errors in tandem walking; turning reflects the number of turning steps and turning time; and Base of Support reflects stride width and its variability. Finally, Global Gait was calculated by summing all gait domains, dividing by the number of gait domains and subsequently calculating a new Z-score [17]. To facilitate interpretation of our findings, we additionally included the two most commonly assessed gait parameters, velocity and cadence.

\section{Statistical analyses}

Differences between persons with and without COPD were studied using the Mann-Whitney U-test for continuous variables and Chi-squared test for categorical variables. Univariate ANCOVAs and linear regression analyses were used to investigate associations of COPD and lung function, respectively, with the gait domains. Age, sex, height, weight, primary education, mini-Mental State Examination (MMSE), pack-years of smoking and use of analgesics (Anatomical Therapeutic Chemical code N02) within 90 days preceding gait assessment were considered potential confounders, and models were adjusted for those showing trends of an association with both COPD and gait $(\mathrm{p}<0.10)$. Models including tandem walk were additionally adjusted for step count and mean step length. We repeated these analyses to investigate associations of lung function and COPD with velocity and cadence.

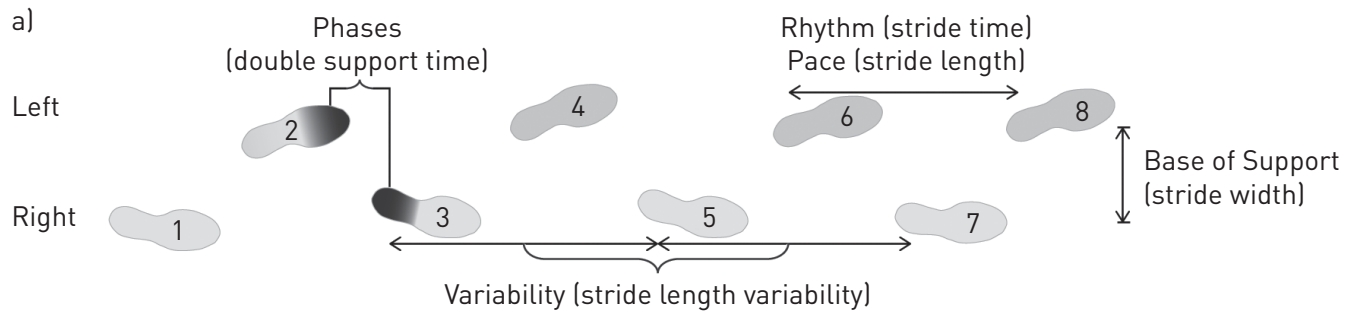

b)

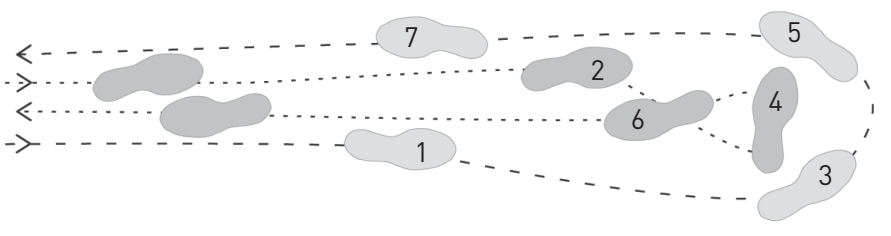

c)

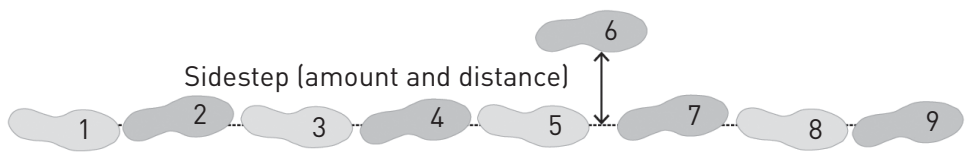

FIGURE 1 The three walking conditions, including the five gait domains of normal walking; a) normal walk; b) turn; c) tandem walk. The gait domains of normal and tandem walking are visualised using constituting gait parameters, which are shown in parenthesis. For turning, only the footsteps used in the calculation of turning time and turning step count are numbered. Turning time was calculated as the time between foot-off of footstep 1 until first contact of footstep 7. Turning step count was calculated as the number of steps in the turn minus one. 
For gait domains that were associated with COPD, we further investigated an association with falls by univariate ANCOVAs.

As cognition has been shown to be very strongly associated with gait, we performed a sensitivity analysis repeating all analyses additionally adjusted for MMSE. In this way, we could evaluate whether any of our associations were the result of residual confounding by cognition.

A p-value below the conventional level of significance $(\mathrm{p}<0.05)$ was considered statistically significant. Statistical analyses were performed using SPSS, version 20.0, for Windows (IBM, North Castle, NY, USA).

\section{Results}

Between March 2009 and December 2011, 1791 participants were invited for gait assessment. Of these, 199 participants did not perform all walking conditions for the following reasons: 152 for physical inability, 36 for technical reasons and 11 for refusal. Of remaining 1592 participants: 182 had to be excluded for technical reasons; 26 for completing $<16$ steps in the normal walks, lowering validity of the measurements [26]; seven for not following instructions; and two for using walking aids. Of the 1375 participants with complete and valid gait data, 1247 had interpretable spirometry data available. Of these, participants with a spirometry suggestive of a restrictive syndrome $(n=46)$ or asthma $(n=107)$ were excluded. Baseline characteristics of the study population $(n=1094)$ are presented in table 1. Among participants, 196 (17.9\%) persons had COPD (96 mild COPD and 100 moderate-to-severe COPD). COPD persons were slightly older, less often female, were more frequently current or former smokers, and presented with worse lung function parameters. All variables considered as potential confounders showed trends of associations with Global Gait or the gait domains.

We found COPD to be associated with lower Rhythm $(-0.21 \mathrm{SD}, 95 \%$ CI $-0.36--0.06 \mathrm{SD}$; table 2) independent of age, sex, height, primary education, pack-years of cigarette smoking and use of analgesics. Regarding COPD severity, the effect size for COPD subjects with frequent exacerbations was four times larger than for COPD subjects without frequent exacerbations $(-0.65 \mathrm{SD}$ (95\% CI $-1.04--0.27 \mathrm{SD})$ compared to -0.16 SD ( $95 \%$ CI $-0.31-0.00 \mathrm{SD}$ ) respectively). Moreover, lower FEV1 was significantly associated with lower Global Gait, Rhythm and Pace (table 2). Although cognitive impairment has an effect on gait, additional adjustment for MMSE hardly changed associations between COPD and gait.

Figure 2 represents the gradual decrease of the age- and sex-adjusted z-scores of walking Rhythm over the 2011 GOLD groups. GOLD groups C and D were significantly related to lower Rhythm, and GOLD group D was also related to lower Global Gait score and Pace, compared with persons without COPD (table E1). In contrast, GOLD group A associated with higher Global Gait and Pace.

We found a very similar pattern of associations for the original gait parameters as for the gait domains. COPD was associated with a lower cadence compared with controls with normal lung function (table 3). Regarding disease severity, more airflow limitation (lower FEV1) associated with slower velocity and

\begin{tabular}{lccc}
\hline TABLE 1 Population characteristics & & \\
Characteristic & No COPD & COPD & p-value $^{\#}$ \\
\hline Subjects $\mathbf{n}$ & 898 & 196 & \\
Age years & $74.2 \pm 5.2$ & $75.5 \pm 5.5$ & 0.003 \\
Females & $483(53.8)$ & $76(38.8)$ & $<0.001$ \\
Height cm & $167.3 \pm 8.9$ & $170.5 \pm 8.9$ & $<0.001$ \\
Weight kg & $76.2 \pm 12.7$ & $80.8 \pm 18.7$ & 0.877 \\
Primary education & $89(10.1)$ & $33(17.1)$ & 0.013 \\
MMSE score & $27.9 \pm 1.8$ & $27.8 \pm 1.9$ & 0.957 \\
Past smokers & $508(56.6)$ & $125(63.8)$ & $<0.001$ \\
Current smokers & $67(7.5)$ & $35(17.9)$ & $<0.001$ \\
Smoking history pack-years & $12.6 \pm 18.3$ & $25.2 \pm 25.9$ & $<0.001$ \\
Taking analgesics & $37(4.1)$ & $14(7.1)$ & 0.051 \\
FEV $\%$ of predicted & $110.9 \pm 16.3$ & $80.8 \pm 18.7$ & $<0.001$ \\
FEV1/FVC \% & $78.6 \pm 4.7$ & $63.4 \pm 6.0$ & $<0.001$ \\
\hline
\end{tabular}

Data are presented as mean \pm SD or $\mathrm{n}(\%)$, unless otherwise stated. COPD: chronic obstructive pulmonary disease; MMSE: mini-Mental State Examination; FEV1: forced expiratory volume in $1 \mathrm{~s}$; FVC: forced vital capacity. \#: for differences between participants with COPD and without COPD, adjusted for age and sex (if applicable). 
TABLE 2 Associations between chronic obstructive pulmonary disease (COPD), forced expiratory volume in $1 \mathrm{~s}$ (FEV1), frequent exacerbations and the gait domains, adjusted for potential confounders

COPD

\begin{tabular}{lc}
\hline Global gait & $0.02(-0.14-0.17)$ \\
Rhythm & $-0.21(-0.36--0.06) *$ \\
Variability & $0.11(-0.05-0.27)$ \\
Phases & $0.12(-0.03-0.28)$ \\
Pace & $-0.05(-0.19-0.08)$ \\
Tandem ${ }^{\#}$ & $0.00(-0.16-0.15)$ \\
Turning & $0.03(-0.13-0.20)$ \\
Base of Support & $0.04(-0.12-0.20)$
\end{tabular}

FEV1 per $10 \%$ predicted

$0.05(0.03-0.08)^{*}$
$0.04(0.01-0.07)^{*}$
$0.00(-0.03-0.03)$
$0.01(-0.02-0.04)$
$0.06(0.04-0.09)^{*}$
$0.03(0.00-0.06)$
$0.00(-0.03-0.03)$
$-0.01(-0.04-0.03)$

Frequent exacerbator

$-0.23(-0.63-0.17)$

$-0.65(-1.04--0.27) *$

$0.26(-0.16-0.68)$

$0.13(-0.28-0.54)$

$-0.30(-0.65-0.05)$

$0.10(-0.32-0.51)$

$-0.04(-0.47-0.39)$

$-0.07(-0.49-0.36)$

Data are presented as differences in z-scores of gait $(95 \% \mathrm{Cl})$. A lower value of gait represents worse gait. All analyses were adjusted for age, sex, height, primary education, pack-years of smoking and use of analgesics. Exacerbations were counted as the total number of moderate and severe exacerbations in the year 2010. Frequent exacerbators were defined as COPD persons having at least two moderate or severe exacerbations. " : additionally adjusted for the step count and step size within the tandem walk. ${ }^{*}: p<0.05$.

cadence. GOLD groups C and D were associated with slower cadence, and GOLD group D was additionally associated with slower velocity (table 3 ).

234 (21.4\%) participants reported that they had fallen in the past 12 months, including 195 (21.7\%) subjects without COPD versus 39 (19.9\%) subjects with COPD ( $\mathrm{p}$-value for difference, 0.564). Figure 3 shows Rhythm to be lowest in COPD persons who fell in the previous year. Fallers with COPD had significantly lower Rhythm than fallers without COPD $(p=0.004)$, nonfallers with COPD $(p=0.022)$ and nonfallers without COPD $(\mathrm{p}<0.001)$, after adjustment for age and sex. These differences remained significant after additional adjustment for height, primary education, pack-years of cigarette smoking and use of analgesics.

\section{Discussion}

In this large, population-based cohort study, we demonstrated that COPD and lung function parameters primarily associate with the temporal aspect of gait. We found COPD to be specifically associated with taking slower steps (lower Rhythm). Moreover, a clinical association between Rhythm and falls according to COPD status was observed. Additionally, we found a higher degree of airflow limitation to associate with worse gait, especially in Rhythm and Pace.

This is the first study to comprehensively investigate the associations of COPD and COPD severity with the gait pattern. A lower walking distance during a 6-min walk or shuttle walk test has been extensively reported in people with COPD; however, literature on gait velocity and other gait deficits is scarce [8,27]. Our results show that COPD is especially related with taking slower steps (lower Rhythm). This indicates that any slowing in gait velocity is mainly driven by a lower cadence. This notion was supported by the associations found with the original gait parameters. A possible explanation for this decrease in cadence may be an

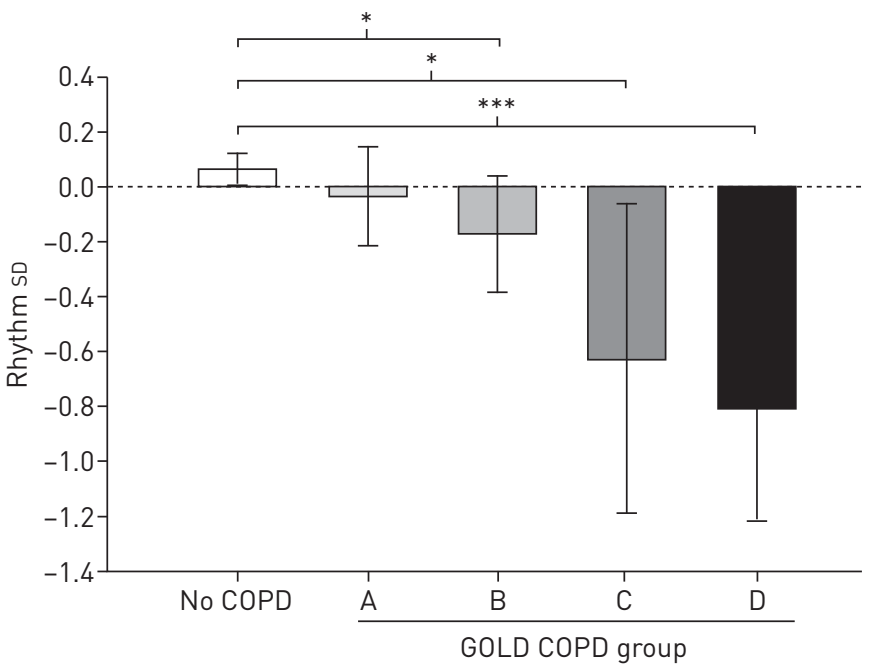

FIGURE 2 Mean walking Rhythm across the 2011 Global Initiative for Chronic Obstructive Lung Disease (GOLD) chronic obstructive pulmonary disease (COPD) groups, adjusted for age and sex. Error bars represent the $95 \%$ confidence interval. ${ }^{*}: \mathrm{p}<0.05$ by ANCOVA; ${ }^{* * *}: \mathrm{p}<0.001$ by ANCOVA. 
TABLE 3 Associations between chronic obstructive pulmonary disease (COPD), forced expiratory volume in $1 \mathrm{~s}$ (FEV1), Global Initiative for Chronic Obstructive Lung Disease (GOLD) groups and original gait parameters, adjusted for potential confounders

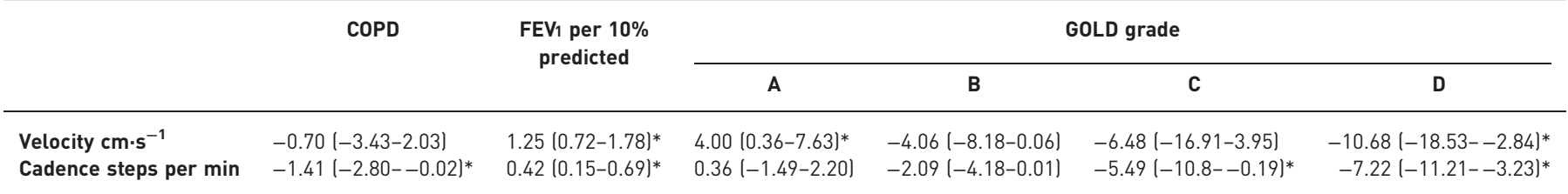

Data are presented as differences in z-scores of gait $(95 \% \mathrm{Cl})$. Lower velocity and cadence can be considered worse. All analyses were

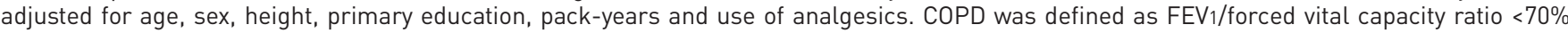
and categorised according to the 2011 GOLD categorisation into group A (low risk and fewer symptoms), B (low risk but more symptoms), C (high risk but fewer symptoms) or D (high risk and more symptoms). *: $p<0.05$.

adaption mechanism to cope with impaired walking endurance due to lack of oxygen supply. Taking slower steps may decrease the oxygen needs of the leg muscles and thus allow for long-distance walking in people with impaired lung function. The lower Rhythm might further result from systemic effects of COPD on the brain, cardiovascular system, muscle strength or bone mineralisation [8, 25, 28-31]. BEAUCHAMP et al. [4] found that gait deficits in patients with COPD were similar to those in patients with an elevated fall risk or neuromuscular disease. Lower limb muscle weakness and impaired activities of daily living are well-established fall risk factors in patients with COPD $[8,32]$. Although gait analysis seems generally the best tool to predict the first fall onset, little is known about the influence of gait deficits on falls in patients with COPD $[8,11]$. Our findings suggest that a low Rhythm might discriminate fallers from nonfallers in subjects with COPD. Because Variability, Tandem, Turning and Base of Support were not significantly different between patients with and without COPD, we could not find evidence for a direct balance disorder in patients with COPD. As the Rhythm correlated less with falling in subjects without COPD, quantitative measures of gait variability may be more sensitive in elderly subjects without COPD [33]. However, further research is needed to determine whether this association of low Rhythm with a history of falling in people with COPD is also reflected in future falling. Several interventions have been described to improve gait regularity, automaticity and gait safety [11]. Based on our results, rhythmic auditory stimulation (RAS)-enhanced gait training, where the cadence of the RAS beats initially matches the initial cadence of patients with COPD and is progressively increased, might be an interesting intervention strategy [34].

The effect of COPD on Rhythm was especially strong in more severe COPD, which is in line with a recent study by YENTES et al. [35]. Similarly, we found the severest GOLD COPD category to associate with worse Global Gait and Pace. In contrast, GOLD A was related to better Global Gait and Phases. This positive association may be the result of initial compensatory mechanisms. Our study also demonstrates that a higher degree of airflow limitation (i.e. lower FEV1) was associated with worse Global Gait, Rhythm and Pace (smaller steps). We found very similar associations with the original gait parameters, supporting our interpretations.

FIGURE 3 Mean walking Rhythm stratified for chronic obstructive pulmonary disease (COPD) and a history of falls, adjusted for age and sex. Error bars represent the 95\% confidence interval. *: $\mathrm{p}<0.05$ by ANCOVA; ${ }^{* *}: \mathrm{p}<0.005$ by ANCOVA; $* * *: p<0.001$ by ANCOVA.

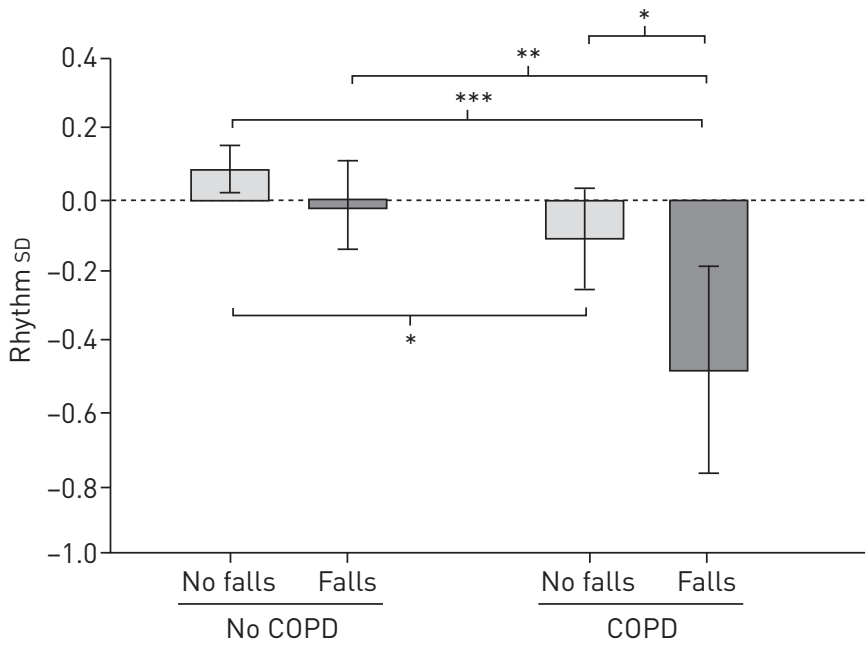


The main strength of our study is that we investigated, for the first time, associations of different gait domains, including different walking conditions, with COPD and lung function in a community-dwelling population. Furthermore, we investigated the influence of disease severity, taking into account exacerbations and the updated GOLD categories besides the severity of airflow limitation on the association of COPD with the gait domains and explored associations of the related gait domain with falling. To cope with confounding, all analyses in our observational study were adjusted for potential confounders that showed a trend of an association with both COPD and gait $[6,19,36]$.

A first potential limitation of this study is that persons with severe COPD and very poor lung function might be less likely to come to the research centre for gait assessment. However, if anything, this selective refusal of participation would have underestimated the strength of association of COPD and worse lung function with gait disturbances. Second, given the cross-sectional analysis and the retrospective interrogation of falls, we cannot infer causality. Consequently, the worse Rhythm in COPD patients may lead to more falls, or falls in COPD patients may aggravate anxiety and unsecured gait, leading to worse Rhythm [6].

In conclusion, COPD is associated with worse gait, especially with taking slower steps, in a community-dwelling population. Additionally, a higher degree of airflow limitation was associated with worse gait, as especially reflected by slower and smaller steps. Further research should investigate the underlying mechanisms of these associations, to enable development of proper intervention strategies to prevent falling in patients with COPD.

\section{Acknowledgements}

The authors thank the study participants, the staff from the Rotterdam Study and the participating general practitioners.

\section{References}

1 Vestbo J, Hurd SS, Agusti AG, et al. Global strategy for the diagnosis, management, and prevention of chronic obstructive pulmonary disease: GOLD executive summary. Am J Respir Crit Care Med 2013; 187: $347-365$.

2 Brusselle GG, Joos GF, Bracke KR. New insights into the immunology of chronic obstructive pulmonary disease. Lancet 2011; 378: 1015-1026.

3 Eisner MD, Blanc PD, Yelin EH, et al. COPD as a systemic disease: impact on physical functional limitations. Am J Med 2008; 121: 789-796.

4 Beauchamp MK, Sibley KM, Lakhani B, et al. Impairments in systems underlying control of balance in COPD. Chest 2012; 141: 1496-1503.

5 Dodd JW, Getov SV, Jones PW. Cognitive function in COPD. Eur Respir J 2010; 35: 913-922.

6 Snijders AH, van de Warrenburg BP, Giladi N, et al. Neurological gait disorders in elderly people: clinical approach and classification. Lancet Neurol 2007; 6: 63-74.

7 Gill TM, Allore HG, Gahbauer EA, et al. Change in disability after hospitalization or restricted activity in older persons. JAMA 2010; 304: 1919-1928.

8 Roig M, Eng JJ, Road JD, et al. Falls in patients with chronic obstructive pulmonary disease: a call for further research. Respir Med 2009; 103: 1257-1269.

9 Kannus P, Sievanen H, Palvanen M, et al. Prevention of falls and consequent injuries in elderly people. Lancet 2005; 366: 1885-1893.

10 Jorstad EC, Hauer K, Becker C, et al. Measuring the psychological outcomes of falling: a systematic review. J Am Geriatr Soc 2005; 53: 501-510.

11 Bridenbaugh SA, Kressig RW. Laboratory review: the role of gait analysis in seniors' mobility and fall prevention. Gerontology 2011; 57: 256-264.

12 Mignardot JB, Deschamps T, Barrey E, et al. Gait disturbances as specific predictive markers of the first fall onset in elderly people: a two-year prospective observational study. Front Aging Neurosci 2014; 6: 22.

13 Abellan van Kan G, Rolland Y, Andrieu S, et al. Gait speed at usual pace as a predictor of adverse outcomes in community-dwelling older people an International Academy on Nutrition and Aging (IANA) Task Force. J Nutr Health Aging 2009; 13: 881-889.

14 Cesari M. Role of gait speed in the assessment of older patients. JAMA 2011; 305: 93-94.

15 Verghese J, Holtzer R, Lipton RB, et al. Quantitative gait markers and incident fall risk in older adults. J Gerontol A Biol Sci Med Sci 2009; 64: 896-901.

16 Studenski S, Perera S, Patel K, et al. Gait speed and survival in older adults. JAMA 2011; 305: 50-58.

17 Verlinden VJ, van der Geest JN, Hofman A, et al. Cognition and gait show a distinct pattern of association in the general population. Alzheimers Dement 2014; 10: 328-335.

18 Watson NL, Sutton-Tyrrell K, Youk AO, et al. Arterial stiffness and gait speed in older adults with and without peripheral arterial disease. Am J Hypertens 2011; 24: 90-95.

19 Verlinden VJ, van der Geest JN, Hoogendam YY, et al. Gait patterns in a community-dwelling population aged 50 years and older. Gait Posture 2013; 37: 500-505.

20 Callisaya ML, Blizzard L, Schmidt MD, et al. A population-based study of sensorimotor factors affecting gait in older people. Age Ageing 2009; 38: 290-295.

21 Hofman A, van Duijn CM, Franco OH, et al. The Rotterdam Study: 2012 objectives and design update. Eur J Epidemiol 2011; 26: 657-686.

22 Celli BR, MacNee W, Agusti A, et al. Standards for the diagnosis and treatment of patients with COPD: a summary of the ATS/ERS position paper. Eur Respir J 2004; 23: 932-946.

23 Macintyre N, Crapo RO, Viegi G, et al. Standardisation of the single-breath determination of carbon monoxide uptake in the lung. Eur Respir J 2005; 26: 720-735. 
24 Rabe KF, Hurd S, Anzueto A, et al. Global strategy for the diagnosis, management, and prevention of chronic obstructive pulmonary disease: GOLD executive summary. Am J Respir Crit Care Med 2007; 176: 532-555.

25 Lahousse L, van den Bouwhuijsen Q, Loth DW, et al. Chronic obstructive pulmonary disease and lipid core carotid artery plaques in the elderly: the Rotterdam Study. Am J Respir Crit Care Med 2013; 187: 58-64.

26 Brach JS, Perera S, Studenski S, et al. The reliability and validity of measures of gait variability in community-dwelling older adults. Arch Phys Med Rehabil 2008; 89: 2293-2296.

27 Karpman C, Depew ZS, LeBrasseur NK, et al. Determinants of Gait Speed in COPD. Chest 2014; 146: 104-110.

28 Bernard S, LeBlanc P, Whittom F, et al. Peripheral muscle weakness in patients with chronic obstructive pulmonary disease. Am J Respir Crit Care Med 1998; 158: 629-634.

29 Lahousse L, Vernooij MW, Darweesh SK, et al. Chronic obstructive pulmonary disease and cerebral microbleeds. The Rotterdam Study. Am J Respir Crit Care Med 2013; 188: 783-788.

30 Lehouck A, Boonen S, Decramer M, et al. COPD, bone metabolism, and osteoporosis. Chest 2011; 139: 648-657.

31 Campos-Obando N, Castano-Betancourt MC, Oei L, et al. Bone mineral density and chronic lung disease mortality: the Rotterdam study. J Clin Endocrinol Metab 2014; 99: 1834-1842.

32 Maltais F, Decramer M, Casaburi R, et al. An official American Thoracic Society/European Respiratory Society statement: update on limb muscle dysfunction in chronic obstructive pulmonary disease. Am J Respir Crit Care Med 2014; 189: e15-e62.

33 Hausdorff JM, Edelberg HK, Mitchell SL, et al. Increased gait unsteadiness in community-dwelling elderly fallers. Arch Phys Med Rehabil 1997; 78: 278-283.

34 Hayden R, Clair AA, Johnson G, et al. The effect of rhythmic auditory stimulation (RAS) on physical therapy outcomes for patients in gait training following stroke: a feasibility study. Int J Neurosci 2009; 119: 2183-2195.

35 Yentes JM, Sayles H, Meza J, et al. Walking abnormalities are associated with COPD: an investigation of the NHANES III dataset. Respir Med 2011; 105: 80-87.

36 Roberts MH, Mapel DW, Hartry A, et al. Chronic pain and pain medication use in chronic obstructive pulmonary disease. A cross-sectional study. Ann Am Thorac Soc 2013; 10: 290-298. 